\title{
Fog Assisted Application Support for Animal Behaviour Analysis and Health Monitoring in Dairy Farming
}

\author{
Mohit Taneja ${ }^{*, \dagger}$, John Byabazaire ${ }^{*, \dagger}$, Alan Davy ${ }^{*, \dagger}$, Cristian Olariu ${ }^{\ddagger}$ \\ *Telecommunications Software and Systems Group, Waterford Institute Of Technology, Waterford, Ireland \\ ${ }^{\dagger}$ CONNECT- Centre for Future Networks and Communications, Ireland \\ $\ddagger$ Innovation Exchange, IBM Ireland, Dublin \\ Email: \{mtaneja, jbyabazaire, adavy\}@tssg.org, cristian.olariu@ie.ibm.com
}

\begin{abstract}
With the exponential growth rate of technology, the future of all activities, including dairy farming involves an omnipresence of widely connected devices. Internet of things (IoT), fog computing, cloud computing and data analytics together offer a great opportunity to increase productivity in the dairy industry. In this paper, we present a fog computing assisted application system for animal behaviour analysis and health monitoring in a dairy farming scenario. The sensed data from sensors is sent to a fog based platform for data classification and analysis, which includes decision making capabilities. The solution aims towards keeping track of the animals' well-being by delivering early warning alerts generated through behavioural analytics, thus aiding the farmer to monitor the health of their livestock and the capability to identify potential diseases at an early stage, thereby also helping in increasing milk yield and productivity. The proposed system follows a service based model, avoids vendor lock-in, and is also scalable to add new features such as the detection of calving, heat, and issues like lameness.

Index Terms-Fog Computing, Cloud Computing, Internet of Things (IoT), Smart Farm, Dairy Farming, Real-time, Data Analytics, Microservices
\end{abstract}

\section{INTRODUCTION}

The Internet of Things (IoT) has reformed the future of connectivity and reachability. While the traditional methods of farming have been more intuitive, the growing demand and supply of agricultural products have made the manual tracking of well-being of the livestock cumbersome and time consuming, and this becomes a major issue with the increase in size and scale of the farm. Over time, the agricultural sector has recognized the need to leverage information and communication technologies (ICT) to improve practice efficiencies, yield, and animal welfare. A smart dairy farm scenario involves a large number of sensors spread across the farm, either in the form of devices worn by the livestock which are used to monitor their health and mobility, or other miscellaneous sensors for measuring farm variables such as soil composition, grass growth, and other environmental scenarios. To ensure proper management for the various processes on the farm, analysis of the data generated by these sensing devices in such a setup becomes of prime importance. Currently, the data collected by these devices is subjected to a cloud based analytics system to gather value in terms of insights and useful information. But this leads to a huge amount of heterogeneous and unstructured data being uploaded to the cloud.

Our work is motivated by the prevalent communication delays [1] observed in primarily cloud based application systems (especially in scenarios with intermittent or no Internet connectivity), thereby affecting responsiveness [2] due to increased latency in getting timely insights in critical usecases; and the fact that the present state of computing systems, applications and architectures inhibit innovation due to the lack of multi-vendor interoperability [3]. In consequence, the alternate directive is to design the systems for pre-processing, with an aim of reducing raw data prior to uploading it to the cloud, and shifting intelligence/analytics closer to the data source. Fog Computing is a relatively new networking paradigm that provides compute, storage and networking resources at the edge of the network. It utilizes the available in-network computing resources and shows the capability of reducing the dependency on the cloud by facilitating data analytics on the network edge [4], thus capable of assisting latencysensitive applications. This improves the responsiveness of the system, reduces resource requirements on the remote cloud infrastructure, and in turn increases the efficiency of the system in terms of energy consumption and network usage [5]. Several interpretations [6] have been proposed for the implementation of fog nodes and their configuration, either via servers, networking devices, cloudlets, base stations, or vehicles. To our understanding, fog node is any compatible device that can be used to deploy applications or a component of an application on it- for example, depending on the specific use-case, it could be a gateway, set-top-box, switch, router, PC, etc. The most suitable configurations to serve as a fog node for such deployments in the particular case of a smart farm scenario include PC, fog servers and compatible networking devices such as gateways.

In this paper, we present a fog computing assisted application system for animal behaviour analysis and health monitoring in a dairy farming scenario. The main contributions of the work are as follows:

- Identification of the farm activities and thus services that demand real-time or near-real time response and decision 
support.

- Design, creation and development of services following a microservices based application design principles to tackle the problem of vendor lock-in, or multi-vendor interoperability.

- Granular service specification such as lameness detection, heat detection, etc. for the farmer/user to choose from, depending upon the individual requirements and size of the farm.

- Scalability and agility to add new services, and provide solutions and features that a user/farmer may demand in future with the usage of the system.

- An added benefit to dairy farmer in case of his/her physical absence from the farm, as the application serves as a medium for other workers to get insights and understand the animals.

The paper is further structured as follows: §II contains motivation, background information and related work, §III contains the architecture and the workflow of the smart dairy farm setup as a part of our real world testbed deployment, §IV presents the animal behaviour analysis and health monitoring informatics from the data thus generated, and $\S \mathrm{V}$ presents the concluding remarks and future work.

\section{Motivation, Background And Related WORK}

With the recent advancements in IoT, the use of computing systems utilizing wireless sensor networks (WSN) has been widely proposed in the agriculture sector in order to facilitate real-time monitoring of farm processes. IoT is an active enabler of smart farming, whereby various entities on the farm can be connected for collecting and exchanging data, thus allowing joint or independent operations. As technology grows to be an integral part of the agricultural and dairy industry, it is important to generate timely insights from the data collected, and enable effective data management. A review by the authors in [7] shows that predictive insights in farming operations drive real-time operational decisions, and redesign business processes for the benefit of various stakeholders in a farming landscape, and that the influence of such systems goes beyond primary production, to the entire supply chain. The authors in [8] provide an implementation of a smart farm setting using a range of environmental sensors and livestock monitoring technologies, while another such implementation of a system for detecting mastitis in dairy cattle and managing their milking processes in the parlour has been presented by the authors in [9]. A study by authors in [10], [11] gives an overview of the sensor systems available for health monitoring of animals in dairy farms. A wireless sensor and actuator based virtual fencing system based on acoustic signals and electric stimuli has been implemented in dairy farms as a replacement of physical barriers to regulate and control mobility of cows within a given boundary in [12].

But a survey in [13] identifies a serious lack of analytics and intelligence in these systems, thus leading to gaps between the desired requirement of the system and proposed solutions. It articulates the need and requirement of intelligence to be present on the premises, in the on-farm systems. Accordingly, the utilization of in-network resources is one of the key factors impacting the intelligence, analytics capacity, and efficiency of the overall system. Another recent survey by authors in [3] identifies the lack of interoperability provided by such systems, and identify the need of developing an integrated system combining edge, fog and cloud to provide application and services. The authors here also identify that technology solutions with no consideration of interoperability results in vendor lock-in, which not only hinders innovation, but also results in higher costs for the farmer/user.

Moreover, fog computing aims for efficient usage of innetwork resources and providing intelligence and decision support faster and closer to source of data [14], [15]. An efficient fog based usage of in-network resources and implementation has been studied by authors in [5], [16]. The suitability of fog computing in context of IoT has been studied by the authors in [17]. While the primary and fundamental insights into data can be used for early event detections, the result of fog analytics can be further sent to the cloud for detailed analysis, and enhancing the learning patterns. The result of the cloud-based historical analysis can then be used to fine-tune and improve the analytics model for fog analytics.

We position our work as an answer to the issues mentioned above, thus bridging the gap, and providing an innovative way that integrates edge, fog and cloud computing to provide a solution specifically in case of smart dairy farming IoT settings. For the reasons mentioned above, we follow a microservices [18] based approach for design, creation and deployment of the application in our setting. This provides many benefits

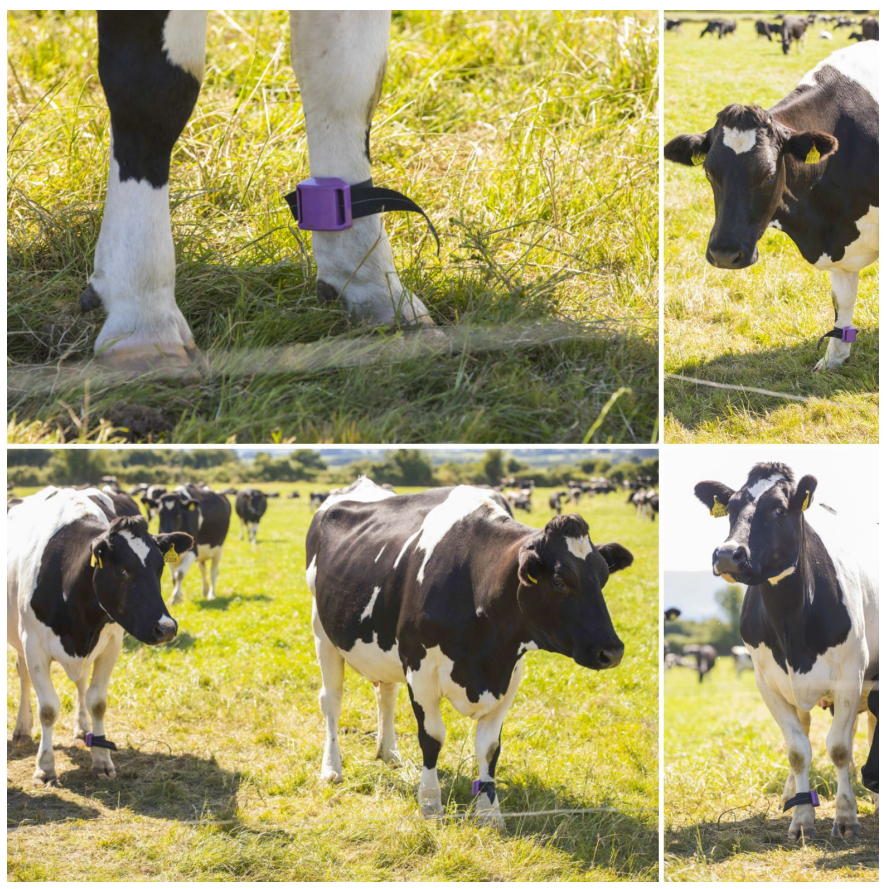

Fig. 1: Pedometer attached as a part of the experiment to the front leg of the cows 
such as better adaptability to technological changes, and more importantly avoids vendor lock-in, i.e. it gives the flexibility to allow simultaneous deployment of software from different vendors implemented using different technologies.

\section{Smart Dairy FARm Setup: ReAl-World Testbed DEPLOYMENT}

The trial ${ }^{1}$ was conducted on a local farm with a full dairy herd of 150 cows in Waterford, Ireland. As a part of the experiment, commercially available Long-Range Pedometers (LRP, ENGS Systems ${ }^{\circledR \circledast}$, Israel) specifically designed for use in dairy cattle were attached to the front leg of cows, as shown in Fig. 1.

\section{A. Architecture}

The overall architecture of the test-bed is shown in Fig. 2. The Pedometer consists of an active system with a (backup) data retention capacity of upto 12 hours that measures the activity of cows (such as standing, lying, walking, etc.) with a sampling frequency of 8 milliseconds, and the thereby generated data unit is sent to the corresponding Receiver and Transceiver in every 6 minutes. The range of the antennas attached to the Receiver and Transceiver is 2 kilometres each, which gives enough coverage to collect data from cows at all times, whether they are grazing in the field, present in their sheds (during adverse weather conditions), or being milked at the milking station.

As shown in Fig. 2, the Receiver is the master unit which sends the received data to the communication unit (RS485 to USB) through wired connection, which in turn then sends it to a local PC (which acts as controller and fog node; configuration $^{2}$ used- Intel ${ }^{\circledR}$ Core $^{\mathrm{TM}}$ 3rd Generation i7-3540M CPU @ 3.00GHz, 16.0 GB RAM, 500 GB Local Storage) through wired connection via USB interface. The collected data is then classified at the fog node itself using a constraint programming [19] based approach into three categories as follows:

- Latency Insensitive Data $\left(\mathbf{L}_{i}\right)$ This includes data that does not require immediate analytics and decision making, and includes the likes of regular logging of cattle data, milking status and related data, soil, water and grass monitoring, etc.

- Latency Sensitive Data $\left(\mathbf{L}_{s}\right)$ This data has a critical value and needs immediate and actionable analysis. This includes activity such as calving alert for pregnant cows. This also includes the streaming data in case of virtual fencing, where the decisions are based on continuous information feeds.

- Latency Tolerant Data $\left(\mathbf{L}_{t}\right)$ This includes data that is usually time insensitive, but gets to the scale of sensitive under certain intervals of time. This includes periodic heat patterns (Estrous Cycle) owing to biological activities.

\footnotetext{
${ }^{1}$ The ethical approval for the experimentation was taken from Research Ethics Committee of Waterford Institute of Technology, Ireland prior to the deployment in July, 2017.

${ }^{2}$ The minimum suggested configuration for the given setup is a Dual Core processor@2.3GHz,4.0 GB RAM, 100 GB local storage.
}

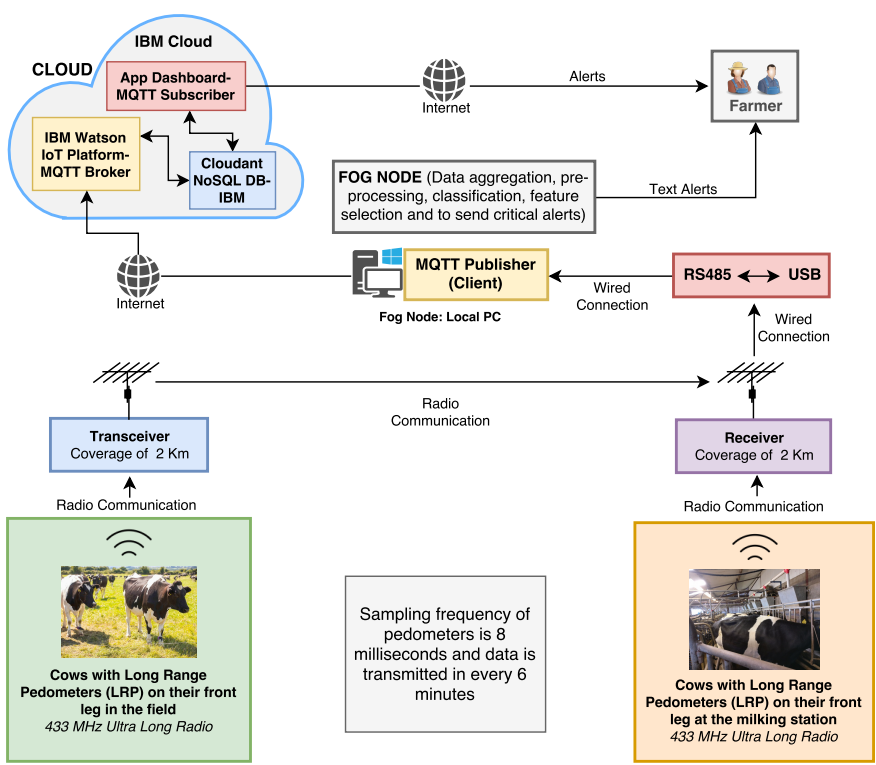

Fig. 2: Overall architecture of the test-bed

While the data categorized as latency insensitive and latency tolerant is given lower priority for immediate fog level computation and might even be provisioned to the cloud, the main point of focus is the immediate attention and actionable decisions on the latency sensitive category. In the case of detection of an event with latency sensitive data, an alert is sent to the farmer. Moreover, we further plan to enhance the data classification approach at fog node in our future work.

We have chosen Message Queue Telemetry Transport (MQTT) [20] as the connectivity protocol between fog node (i.e local PC) and cloud (service instances running on IBM Cloud) in our deployment setting. MQTT is an open-source protocol originally invented and developed by IBM [21]. It is a lightweight publish-subscriber model based protocol designed on top of the TCP/IP stack. It is specifically targeted for remote location connectivity with characteristically unreliable network environments such as high delays and low bandwidth [22], which is one of the issues in remote farm based deployments such as ours.

The MQTT architecture comprises of two components, namely MQTT clients (such as publishers and subscribers) and MQTT broker (for mediating messages between publishers and subscribers). In our setup these components are as follows:

- MQTT Publisher: Script running on fog node (i.e local $\mathrm{PC}$ at farm)

- MQTT Broker: IBM Watson IoT Platform (as a service on IBM Cloud)

- MQTT Subscriber: Application designed and hosted on IBM Cloud

Thus, the data from fog node after classification as described above is streamed to IBM Waston IoT platform using MQTT; the IBM Watson IoT platform receives all these messages, and the MQTT subscriber listening to the events of this broker 


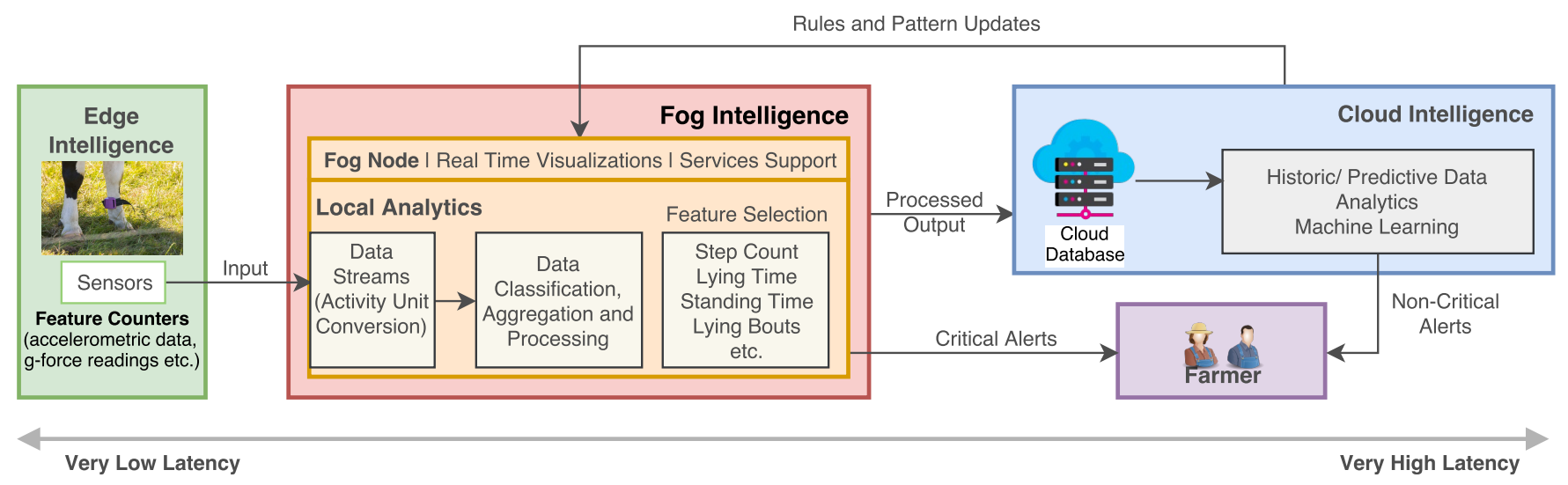

Fig. 3: Work flow and data flow in the testbed deployment

picks up all the data and stores it in Cloudant NoSQL JSON Database (Database service on IBM Cloud).

\section{B. Work Flow}

The detailed work flow of the experimental setup is as shown in Fig. 3. The fog node also provides a dashboard for the farmer and serves as a visual medium to see the event information and other related sensor data. After gathering initial insights from the collected data and generating the corresponding alert/response, the aggregated, processed data is sent to the cloud for historical storage and analysis. The cloud is also the site for fusion of the data from other sources, such as weather data. The learning pattern from historical data analysis at cloud is sent back to fog node for further enhancement of the system, and to increase the overall efficiency and responsiveness. All the long-term information and data is stored in the cloud, and like the fog, it too provides a dashboard where the farmer can input and modify any relevant information related to their livestock, and demand further features and services.

We envision fog as a way to do things better in cloud. Thus, to sum it up, the fog node serves the following purposes:

- Acts as MQTT publisher to the cloud

- Real-time local visualization medium, and a platform for performing analytics

- Performs local aggregation and filtering of data, and sends only high-valued data to cloud for historical storage and analytics

\section{Animal Behaviour Analysis and Health Monitoring From the Data Generated}

As a part of the experiment, we collected the data of 150 cows for a period of 30 days spanning July and August 2017, and analysed it to understand the behaviour of the

TABLE I: Age Distribution of Cows in the Herd

\begin{tabular}{|c|c|c|c|c|c|c|c|c|c|c|}
\hline Age (in years) & 2 & 3 & 4 & 5 & 6 & 7 & 8 & 9 & 10 & 11 \\
\hline Number of Cows & 30 & 33 & 27 & 21 & 8 & 7 & 6 & 7 & 9 & 2 \\
\hline
\end{tabular}

herd. As the number of cows being analysed are significant in number, an age based analysis as plotted is more insightful than an individual analysis for deriving behavioural patterns. The distribution of the number of cows by age on the said farm is as shown in TABLE I. Fig. 4 shows a group of box plots that represent the activity of cows in the duration of the data sampled for analysis, which includes: (a) the total amount of hours spent lying, (b) the total amount of time spent standing/grazing, (c) the count of the total number of times the position changed from standing to lying, and (d) the number of steps taken. At the time of the analysis, 140 of these cows were between 1.5 to 3 months into their pregnancy. As inferred from the plots, ideally, if left in pastures on their own accord, the cow could spend a third of its time grazing, a third of its time chewing the cud, and the remaining third sleeping. But these numbers vary greatly depending on breed, size, age, and activity level. Being ruminants, cows spend a significant time in the process of rumination, commonly referred to as chewing the cud, which is an important part of the digestive process and is a major part of day lying time. Fig. 5 shows the lying behaviour of both young (age 2-5) and old (age 612) cows throughout the day averaged over 30 days, which owes for observations such as the fact that all cows had an increase in lying time after the milking sessions, and that young cows begin their day almost an hour earlier, and are more active throughout the day. The lying time of the cow thus is cumulative of rumination, sleep, rest, and other factors, which include state of pregnancy, medical conditions, range of illnesses, and potential lameness. As these activities are largely affected/dependant on seasonal weather conditions as well as individual habits of the cows, thus training the system to the herd's behaviour for a longer time would help predict conditions like lameness and analyse any abnormal behaviour to a potential illness. The outliers in the plots may mean that something is wrong with the said cow, but the same cannot be predicted absolutely without prolonged behavioural analysis and continued health monitoring. Although, until the system learns from the individual behavioural patterns and history, any outliers and anomalies are reported to the farmer as early 


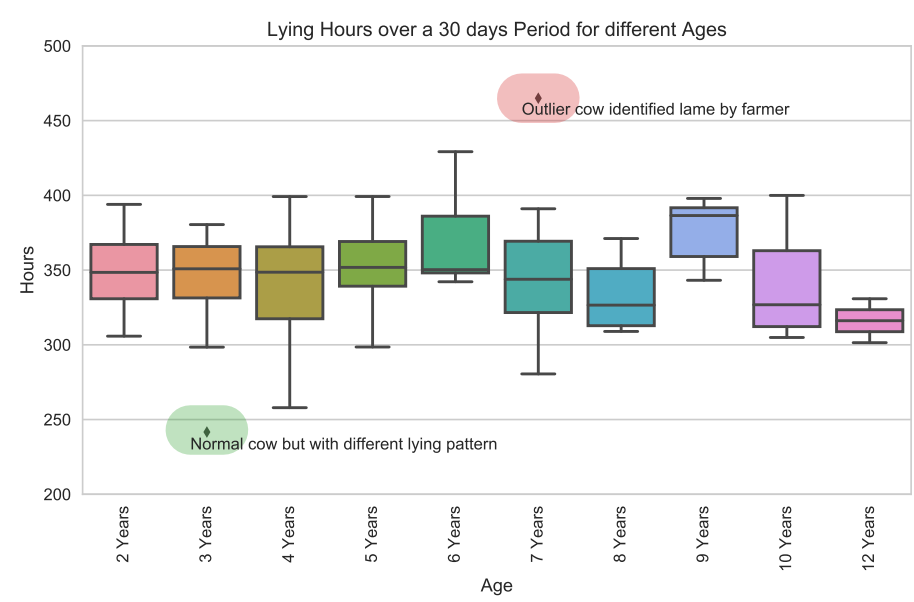

(a) The total amount of hours spent lying

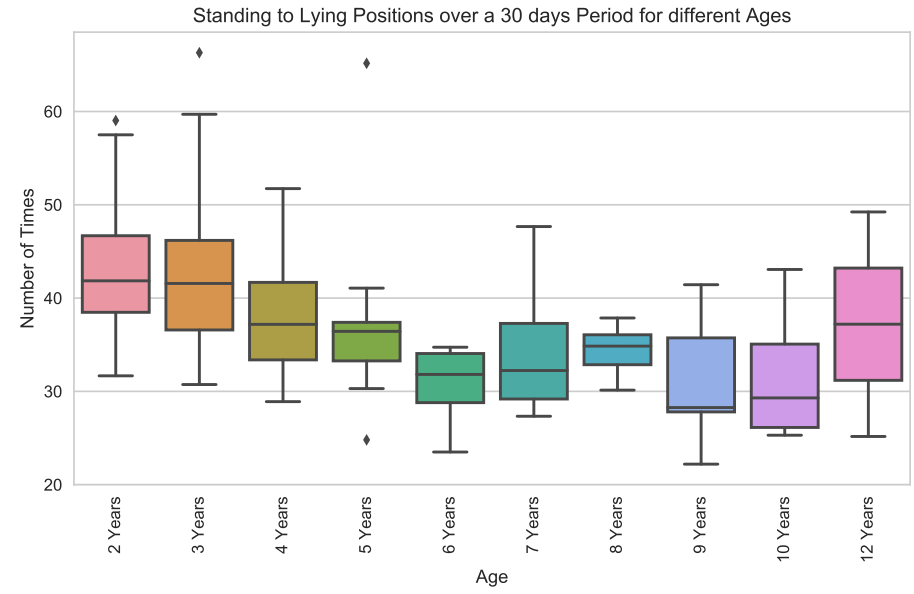

(c) The count of the total number of times the position changed from standing to lying

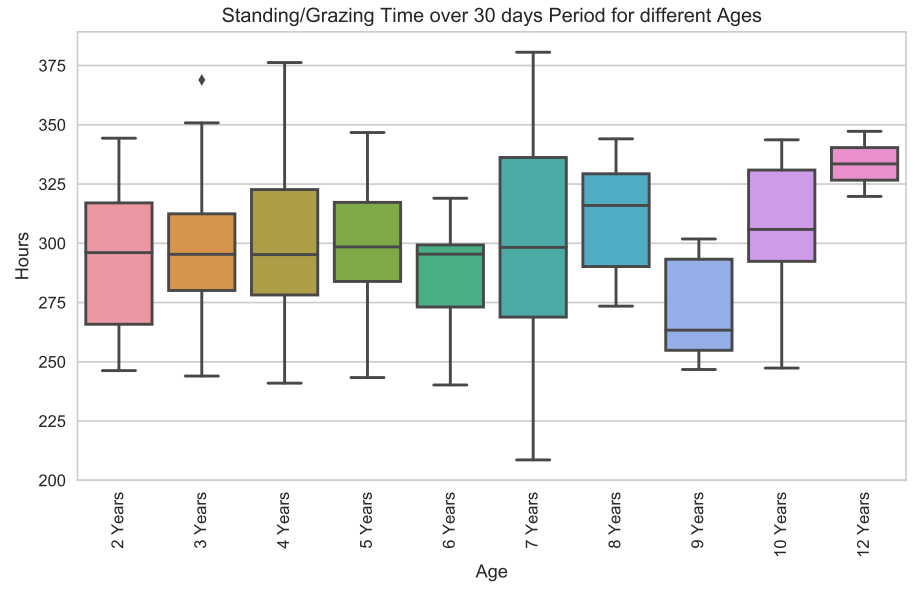

(b) The total amount of time spent standing/grazing

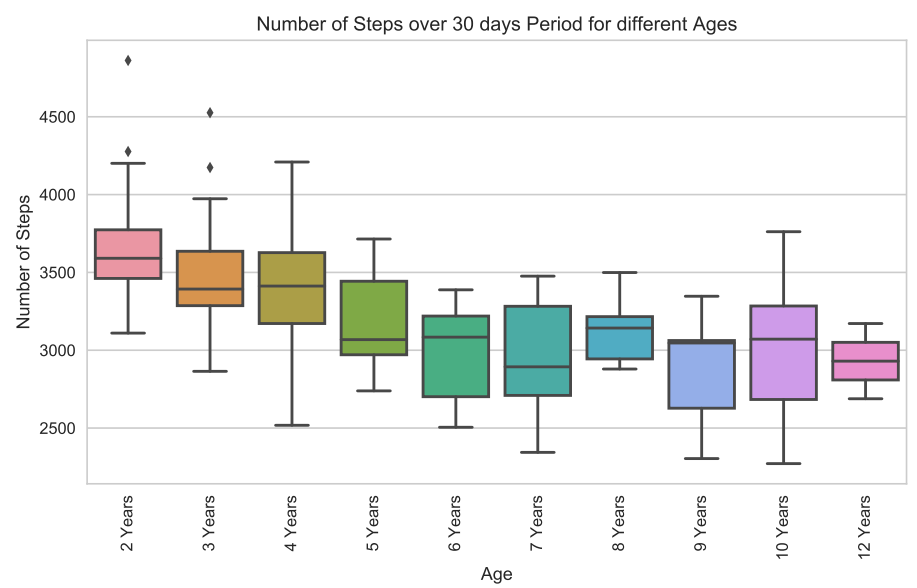

(d) The number of steps taken

Fig. 4: The activity of the cows as noticed in 30 days of analysis. While outliers in the plot may mean something is wrong with the cow, it is not absolute. For example in Fig. 4a, the outlier cow highlighted in red was identified lame by the farmer, but this is not the case for all cows. The one highlighted in green in the same plot was confirmed to be perfectly healthy even though its lying and standing/grazing behaviour has been completely different from the rest.

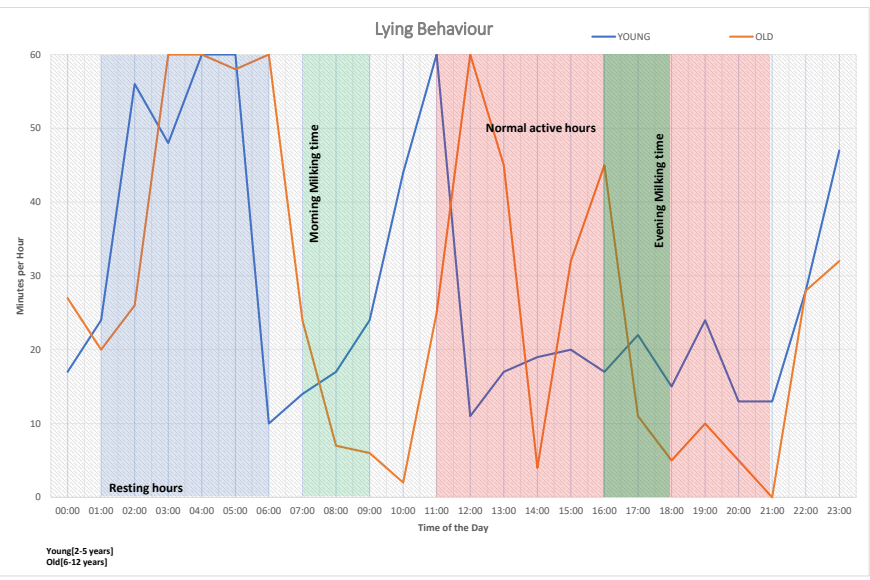

Fig. 5: Daily lying pattern of young (age 2-5) and old (age 6-12) cows averaged over a period of 30 days. warning alerts. A further analysis of such behavioural changes as a result of health related issues will be the basis of our next work.

\section{Real World DePloyment: LEARNings AND PRACTICAL EXPERIENCES}

The primary challenge is to design an IoT solution to meet the specified objective given the highly variable, harsh and resource constrained environment in a smart dairy farming setting. This includes making the system resilient and fault tolerant to cope up with the variable farm environments, including weather based network outages and connectivity issues because of remote location of the farm. The use of fog computing brings efficiency and sustainability to the overall IoT solution being proposed.

Practical implementation of an end-to-end IoT solution towards a specified objective is a complex and intricate process. 
With respect to our real world deployment, deciding which sensors to choose to build the sensing infrastructure, the right application protocols, using best software development practices - the overall solution demands a dedicated brainstorming for a reasonable period of time to achieve efficiency and sustainability. The tradeoff between initial costs for the setup of such a system would be balanced in the long term not just with payback via advantages, but also potential savings, including reduced cost of connectivity to the cloud, low bandwidth requirements, extended battery life cycles on sensors, etc.

\section{CONCLUSION AND Future WORK}

The results suggest that such a fog computing based application support has several advantages over the traditional cloud centric system, and provides real-time data analytics support with increased efficiency. To successfully operate any farm, effective livestock management is imperative. Efficient, affordable, and scalable application support for livestock management thus plays a progressively important role in modern dairy farming, and demand for such systems is increasing in agriculture sector. This becomes particularly more important with the increasing size and scale of a farm and its activities. Our results suggest that the designed system supports all the above requirements along with the agility and scalability to add further features if required, while allowing for multivendor interoperability due to the microservices approach. In our future work, we aim to extend the behavioural analytics and provide microservices specific to certain cow management problems such as lameness. From our current investigation, we found that lameness is a severe health problem in dairy cattle that demands real-time data analytics and decision support, and we aim to develop a machine learning based method for early detection of lameness in dairy cattle and include it as a service in the application developed in our current work.

Lastly, the collected data from the real world deployment is available to be shared with the academic research community upon request.

\section{ACKNOWLEDGEMENT}

This work has emanated from research conducted with the financial support of Science Foundation Ireland (SFI) and is co-funded under the European Regional Development Fund under Grant Number 13/RC/2077.

\section{REFERENCES}

[1] A. H. Ngu, M. Gutierrez, V. Metsis, S. Nepal, and Q. Z. Sheng, "Iot middleware: A survey on issues and enabling technologies," IEEE Internet of Things Journal, vol. 4, no. 1, pp. 1-20, Feb 2017.

[2] A. Botta, W. de Donato, V. Persico, and A. Pescap, "Integration of cloud computing and internet of things: A survey," Future Generation Computer Systems, vol. 56, pp. 684 - 700, 2016. [Online]. Available: http://www.sciencedirect.com/science/article/pii/S0167739X15003015

[3] A. Jukan, X. Masip-Bruin, and N. Amla, "Smart computing and sensing technologies for animal welfare: A systematic review," ACM Comput. Surv., vol. 50, no. 1, pp. 10:1-10:27, Apr. 2017. [Online]. Available: http://doi.acm.org/10.1145/3041960
[4] F. Bonomi, R. Milito, J. Zhu, and S. Addepalli, "Fog computing and its role in the internet of things," in Proceedings of the First Edition of the MCC Workshop on Mobile Cloud Computing, ser. MCC ' 12. New York, NY, USA: ACM, 2012, pp. 13-16. [Online]. Available: http://doi.acm.org/10.1145/2342509.2342513

[5] M. Taneja and A. Davy, "Resource aware placement of iot application modules in fog-cloud computing paradigm," in 2017 IFIP/IEEE Symposium on Integrated Network and Service Management (IM), May 2017, pp. $1222-1228$.

[6] R. Mahmud and R. Buyya, "Fog computing: A taxonomy, survey and future directions," CoRR, vol. abs/1611.05539, 2016. [Online]. Available: http://arxiv.org/abs/1611.05539

[7] S. Wolfert, L. Ge, C. Verdouw, and M.-J. Bogaardt, "Big data in smart farming a review," Agricultural Systems, vol. 153, pp. $69-80$, 2017. [Online]. Available: http://www.sciencedirect.com/science/article/ pii/S0308521X16303754

[8] K. Taylor, C. Griffith, L. Lefort, R. Gaire, M. Compton, T. Wark, D. Lamb, G. Falzon, and M. Trotter, "Farming the web of things," IEEE Intelligent Systems, vol. 28, no. 6, pp. 12-19, Nov 2013.

[9] M.-C. Chen, C.-H. Chen, and C.-Y. Siang, "Design of information system for milking dairy cattle and detection of mastitis," Mathematical Problems in Engineering, vol. 2014, p. 19, 2014.

[10] W. Steeneveld and H. Hogeveen, "Characterization of dutch dairy farms using sensor systems for cow management," Journal of Dairy Science, vol. 98, no. 1, pp. 709 - 717, 2015. [Online]. Available: http://www.sciencedirect.com/science/article/pii/S0022030214007863

[11] I. Andonovic, C. Michie, M. Gilroy, H. G. Goh, K. H. Kwong, K. Sasloglou, and T. Wu, Wireless Sensor Networks for Cattle Health Monitoring. Berlin, Heidelberg: Springer Berlin Heidelberg, 2010, pp. 21-31. [Online]. Available: https://doi.org/10.1007/978-3-642-10781-8_ 3

[12] T. Wark, D. Swain, C. Crossman, P. Valencia, G. Bishop-Hurley, and R. Handcock, "Sensor and actuator networks: Protecting environmentally sensitive areas," IEEE Pervasive Computing, vol. 8, no. 1, pp. 3036, Jan 2009

[13] C. Rutten, A. Velthuis, W. Steeneveld, and H. Hogeveen, "Invited review: Sensors to support health management on dairy farms," Journal of Dairy Science, vol. 96, no. 4, pp. 1928 - 1952, 2013. [Online]. Available: http://www.sciencedirect.com/science/article/pii/S0022030213001409

[14] F. Bonomi, R. Milito, P. Natarajan, and J. Zhu, Fog Computing: A Platform for Internet of Things and Analytics. Cham: Springer International Publishing, 2014, pp. 169-186. [Online]. Available: https://doi.org/10.1007/978-3-319-05029-4_7

[15] M. Taneja and A. Davy, "Resource aware placement of data analytics platform in fog computing," Procedia Computer Science, vol. 97, no. Supplement C, pp. 153 - 156, 2016, 2nd International Conference on Cloud Forward: From Distributed to Complete Computing. [Online]. Available: http://www.sciencedirect.com/science/ article/pii/S1877050916321111

[16] M. Taneja and A. Davy, "Poster abstract: Resource aware placement of data stream analytics operators on fog infrastructure for internet of things applications," in 2016 IEEE/ACM Symposium on Edge Computing (SEC), Oct 2016, pp. 113-114.

[17] S. Sarkar, S. Chatterjee, and S. Misra, "Assessment of the suitability of fog computing in the context of internet of things," IEEE Transactions on Cloud Computing, vol. PP, no. 99, pp. 1-1, 2015.

[18] B. Butzin, F. Golatowski, and D. Timmermann, "Microservices approach for the internet of things," in 2016 IEEE 21st International Conference on Emerging Technologies and Factory Automation (ETFA), Sept 2016, pp. $1-6$

[19] F. Rossi, P. v. Beek, and T. Walsh, Handbook of Constraint Programming (Foundations of Artificial Intelligence). New York, NY, USA: Elsevier Science Inc., 2006.

[20] "MQTT," http://mqtt.org/, (Last Accessed on August 03,2017).

[21] "Getting to know MQTT," https://www.ibm.com/developerworks/ library/iot-mqtt-why-good-for-iot/index.html, (Last accessed on August 03,2017).

[22] S. Lee, H. Kim, D. k. Hong, and H. Ju, "Correlation analysis of mqtt loss and delay according to qos level," in The International Conference on Information Networking 2013 (ICOIN), Jan 2013, pp. 714-717. 